Western University

Scholarship@Western

Brain and Mind Institute Researchers'

Publications

Brain and Mind Institute

8-1-2001

Discrimination of computer-graphic stimuli by mice: a method for the behavioral characterization of transgenic and gene-knockout models.

\author{
T J Bussey \\ L M Saksida \\ L A Rothblat
}

Follow this and additional works at: https://ir.lib.uwo.ca/brainpub

Part of the Neurosciences Commons, and the Psychology Commons

Citation of this paper:

Bussey, T J; Saksida, L M; and Rothblat, L A, "Discrimination of computer-graphic stimuli by mice: a method for the behavioral characterization of transgenic and gene-knockout models." (2001). Brain and Mind Institute Researchers' Publications. 88.

https://ir.lib.uwo.ca/brainpub/88 


\title{
Discrimination of Computer-Graphic Stimuli by Mice: A Method for the Behavioral Characterization of Transgenic and Gene-Knockout Models
}

\author{
Timothy J. Bussey and Lisa M. Saksida \\ University of Cambridge
}

\author{
Lawrence A. Rothblat \\ George Washington University
}

\begin{abstract}
An automated method is described for the behavioral testing of mice in an apparatus that allows computer-graphic stimulus material to be presented. Mice responded to these stimuli by making a nose-poke toward a computer monitor that was equipped with a touchscreen attachment for detecting responses. It was found that $\mathrm{C} 57 \mathrm{BL} / 6$ mice were able to solve single-pair visual discriminations as well as 3-pair concurrent visual discriminations. The finding that mice are capable of complex visual discriminations introduces the possibility of testing mice on nonspatial tasks that are similar to those used with rats, monkeys, and humans. Furthermore, the method seems particularly well suited to the comprehensive behavioral assessment of transgenic and gene-knockout models.
\end{abstract}

Over the past decade, the mouse has become one of the most useful models for studying the cellular mechanisms underlying learning and memory. A number of emerging transgenic and gene-knockout technologies are now available that can be used to help answer fundamental questions regarding the brain structures involved in learning and the molecular basis of the plastic changes that occur in these structures during the formation of new memories (Mayford \& Kandel, 1999; Silva, Kogan, Frankland, \& Kida, 1998). In addition, mouse models are being generated that replicate many of the pathological features of brain disorders, such as the $\beta$-amyloid deposits that are characteristic of Alzheimer's disease (e.g., Hsiao et al., 1996). Already, these advances have created an impressive body of data that has substantially furthered researchers' understanding of the way genetic modifications can affect higher cognitive functions.

It is widely acknowledged, however, that despite these advances, behavioral paradigms for measuring learning and memory in mice remain somewhat limited, focusing, for the most part, on tasks that measure spatial ability, such as the Barnes maze (Barnes, 1979) and the Morris water maze (Morris, 1984), and tasks that measure simple forms of associative learning, such as classically conditioned fear (Kim \& Fanselow, 1992; LeDoux, 1992). A1though data from such techniques are clearly informative, a primary goal of using animal models is to facilitate comparison between animal data and data collected from human patients and controls. Therefore, the cognitive tasks used to study animals should match as closely as possible those used to study human

Timothy J. Bussey and Lisa M. Saksida, Department of Experimental Psychology, University of Cambridge, Cambridge, England; Lawrence A. Rothblat, Department of Psychology, George Washington University.

This research was supported by the Janet W. Johnson Fund of the Department of Psychology, George Washington University.

Correspondence concerning this article should be addressed to Lawrence A. Rothblat, Department of Psychology, George Washington University, 2125 G Street, NW, Washington, DC 20052. Electronic mail may be sent tolar@gwu.edu. populations. For this reason, the studies of learning and memory in other animal models (e.g., monkeys) predominantly use visual object discrimination tasks. The validity of these tasks can be assessed by comparing the performance of animals with the performance of human patients with amnesia on analogous cognitive tests. Object discrimination tasks have been successfully adapted for use with rats (e.g., Rothblat \& Hayes, 1987; Rothblat, Vnek, Gleason, \& Kromer, 1993) and are now being implemented using computer-automated techniques (Bussey, Muir, \& Robbins, 1994; Gaffan \& Eacott, 1995). This newer procedure, which allows for the presentation of computer-graphic stimuli, further facilitates the comparison between animal studies and human studies and capitalizes on the many advantages of automated testing.

In the present study, we explored the potential of a computerautomated touchscreen testing method for the mouse. This method is similar to a technique described by Bussey et al. (1994) for use with the rat, which has been subsequently applied to the study of the effects of discrete brain damage in this species (e.g., Bussey, Muir, Everitt, \& Robbins, 1997; Bussey, Warburton, Aggleton, \& Muir, 1998). Here, we report the performance of 8 normal C57BL/6 mice on several visual discriminations, including a threepair concurrent visual discrimination.

\section{Method}

\section{Subjects}

Eight male C57BL/6 mice (Jackson Laboratory, Bar Harbor, ME), approximately 60 days old at the start of testing, served as subjects. Throughout the duration of the study, the mice were individually housed in plastic cages and were maintained on a level of Lab Chow (Ralston-Purina, St. Louis, MO) that was sufficient to maintain their body weight at $80-85 \%$, adjusted for growth.

\section{Apparatus and Procedures}

The apparatus (see Figure 1A) and testing methods used for visual discrimination training were similar to those previously described (Bussey 


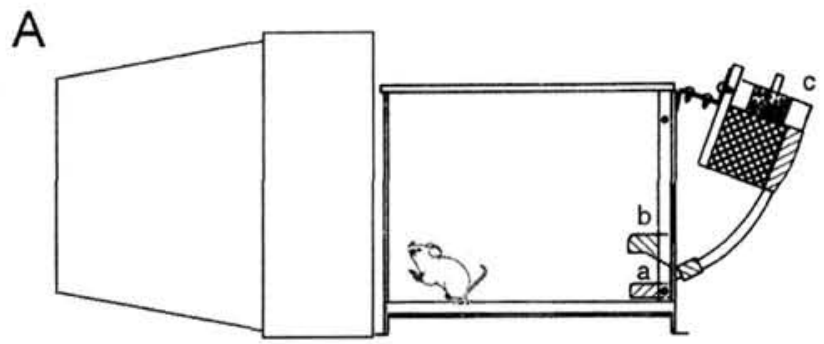

B Single-Pair

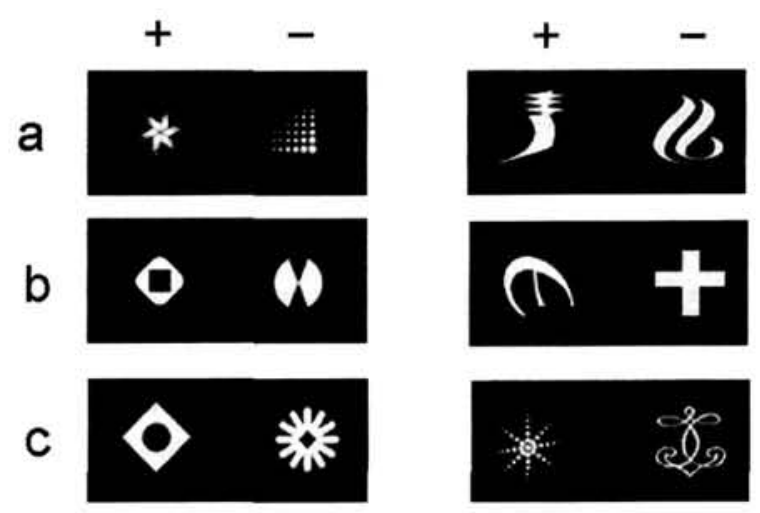

Figure I. A: Apparatus used for behavioral testing of mice. $\mathrm{a}=$ food cup; $\mathrm{b}=$ initiate lever; $\mathrm{c}=$ pellet dispenser. $\mathrm{B}$ : Pairs of stimuli $(\mathrm{a}, \mathrm{b}$, and $\mathrm{c})$ used in the three single-pair discrimination tasks. C: The three pairs of stimuli used in the three-pair concurrent discrimination task. In all tasks, a stimulus appeared either on the left or on the right, determined pseudorandomly in each trial. The rewarded $(+)$ and unrewarded $(-)$ stimulus in each pair are indicated.

et al., 1994; Rothblat, Schwartz, \& Kasdan, 1978). Briefly, the mice were tested in a Plexiglas chamber $(20 \times 20 \times 20 \mathrm{~cm})$. A food well $(4 \times 3 \times 2$ $\mathrm{cm}$ ) was attached to an externally placed pellet dispenser (Model ENV 203-20, MED Associates, St. Albans, VT) and was located on the center of the wall at the rear of the chamber. The initiate lever $(5 \times 1.5 \times 1 \mathrm{~cm})$ was positioned $2.5 \mathrm{~cm}$ above the top of the food well. A video display unit (VDU), on which the stimuli were presented, was placed at the other end of the chamber. Attached to the front of the VDU was a touchscreen unit (Model 3457, CarrollTouch International, Tokyo, Japan). The touchscreen, which has an array of horizontally and vertically placed photocells, was used to detect the location of nose pokes to the VDU. Because the touchscreen photocells were located approximately $1 \mathrm{~cm}$ from the VDU screen, the mice were not required to make contact with the VDU, only to come very close to touching it. The stimuli used in the tasks consisted of approximately equiluminescent white patterns (approximately $4 \times 4 \mathrm{~cm}$ ) presented on a black background (see Figure IB and IC). The bottom of each stimulus was located approximately $3 \mathrm{~cm}$ from the floor. The apparatus was controlled and monitored by an IBM-compatible PC and used programs written in Microsoft QuickBASIC.

We acclimated the mice ( 20 min per day) by placing each in the test apparatus and allowing access to food pellets $(20 \mathrm{mg}$. P.J. Noyes, Lancaster, $\mathrm{NH}$ ) in the food well at the rear of the chamber (Phase 1). Phase 1 continued until the mice were eating freely from the food well and consuming 20 pellets within $30 \mathrm{~min}$ (range $=3-5$ sessions). To pretrain the mice to respond to the VDU display (Phase 2), we pseudorandomly presented a white square ( 20 trials per day) an equal number of times on the left and right side of the display screen. The square remained on the screen until the mouse responded to it, after which the mouse was rewarded with a food pellet and the presentation of a tone. The square reappeared on the screen after a 10-s interval. Mice continued in Phase 2 until they completed the 20-trial session within $30 \mathrm{~min}$ (range $=4-6$ sessions). Phase 3 of pretraining, which required pressing the initiate lever to produce stimulus onset, was also continued until the mice completed 20 trials within $30 \mathrm{~min}$. Because the mice readily climbed on and depressed the initiate lever, the trial-initiate component of the shaping procedure was rapidly acquired, and the mice typically completed Phase 3 in 2 or 3 sessions.

Discrimination training was similar to Phase 3 of pretraining because the mice were required to initiate each trial by depressing the lever to produce stimulus onset. However, in the discrimination training, two different visual stimuli appeared on the VDU. One of the stimuli was designated as the rewarded stimulus, whereas the other was designated as the unrewarded stimulus (see Figure 1B). The mouse approached the VDU and selected one of the two stimuli by responding to it directly with a nose poke. Correct responses were followed by the disappearance of the stimuli, the presentation of a tone, and the delivery of a food pellet. Initiation of the next trial would then occur $5 \mathrm{~s}$ after the response. An incorrect response terminated the stimuli and deactivated the initiate lever for $20 \mathrm{~s}$. On the single-pair discrimination tasks, the same pair of stimuli appeared on all trials. The left-right position of the correct stimulus was varied pseudorandomly with the stipulation that the correct stimulus appeared in each position on $50 \%$ of the trials. Subjects were tested for 20 trials per day with a correction procedure that repeated the same stimulus presentation until the mouse responded correctly. The criterion for single-pair discrimination problems was $90 \%$ correct responses excluding correction trials (i.e., at least 18 out of 20 correct first-choice trials). All 8 mice were trained to criterion on the first single-pair problem (Discrimination A; Figure 1B, panel a). Half of the mice (Cohort 1 ) were then trained to discriminate a more difficult pair of stimuli (Discrimination B; Figure IB, panel b), whereas the other 4 mice (Cohort 2) learned a different difficult single-pair problem (Discrimination C; Figure 1B, panel c). The mice in Cohort 1 were subsequently trained on a three-pair concurrent discrimination (see Figure 1C) in which 24 trials were presented in each daily session. For the concurrent task, the mice were required to learn three separate single-pair discriminations simultaneously. Following trial initiation, one of the three pairs of stimuli appeared on the VDU. Each of the three pairs of stimuli was presented eight times during a session in an intermingled fashion. Throughout the concurrent training, the same member of each stimulus pair was rewarded. Each mouse was tested for 50 sessions.

Following acquisition training, all mice were retrained on the previously learned single-pair discriminations to measure retention. Thus, after completing the three-pair concurrent task, mice in Cohort 1 were retrained on Discrimination B. In this case, the retention interval was approximately 10 weeks. Mice in Cohort 2 were retrained on Discrimination $\mathrm{C}$ after a retention interval of 1 week, during which time the mice were not tested. The testing procedure for retraining was the same as that for initial acquisition.

\section{Results}

\section{Acquisition of Visual Discrimination Tasks}

As shown in Figure 2A, all 8 mice were able to discriminate the computer-graphic stimuli used in Discrimination A when presented on the touchscreen testing apparatus. The number of sessions required by the mice to attain the criterion of 18 out of 20 correct responses ranged between 8 and 19. The mean number of sessions it took the mice to reach this criterion was 11.5 , with a standard error of 1.32. We obtained a similar pattern of data on the more difficult Discriminations B and C by Cohorts 1 and 2, 

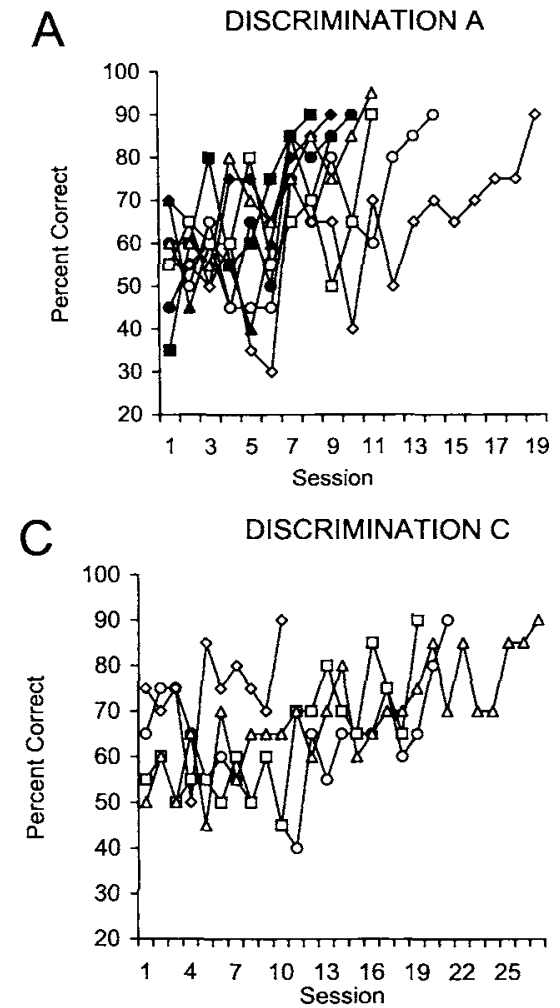

B

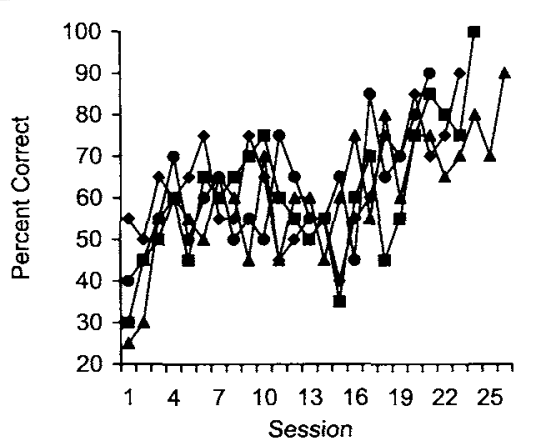

D

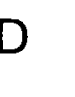

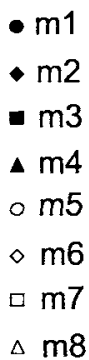

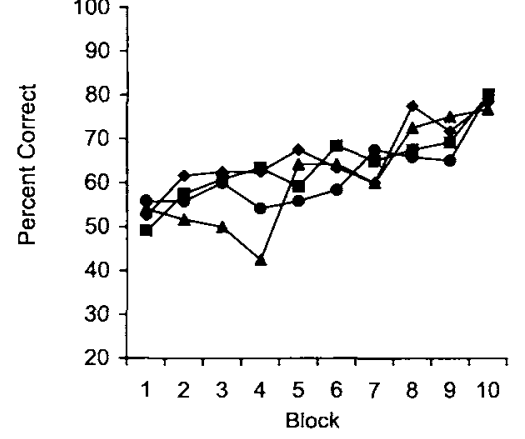

Figure 2. Acquisition curves for each of the 8 mice on the four tasks examined. A: Performance of all 8 mice on Discrimination A. B: Performance of mice in Cohort 1 on Discrimination B. C: Performance of mice in Cohort 2 on Discrimination C. D: Performance of mice in Cohort 1 on a three-pair concurrent discrimination task. $\mathrm{m}=$ mouse.

respectively. These data are shown in Figures $2 \mathrm{~B}$ and $2 \mathrm{C}$. For Discrimination $B$, the number of sessions required by the mice to attain a criterion of 18 out of 20 correct responses ranged between 21 and 26 . The mean number of sessions it took to reach this criterion was 23.5 , with a standard error of 1.20 . For Discrimination $\mathrm{C}$, the number of sessions required to attain this criterion ranged between 10 and 27 . The mean number of sessions it took to reach this criterion on Discrimination $C$ was 19.3 , with a standard error of 4.06 .

As shown in Figure 2D, all 4 mice in Cohort 1 were able to acquire a three-pair concurrent visual discrimination task, and there was very little variability in their scores. The mice averaged $79 \%$ correct responses over the final five sessions (Block 10) of the concurrent task. All of the mice reached $85 \%$ correct responses in at least one session.

\section{Retention of Visual Discrimination Tasks}

The mean number of sessions required by the mice in Cohort 1 to relearn Discrimination B was 6.3, with a standard error of 0.73 . This compares with the mean acquisition score of $23.5( \pm 1.20)$. On the first day of retesting, the mice in Cohort 1 achieved a mean of 13.5 ( \pm 0.75 ) correct responses, for an average accuracy of $68 \%$. To evaluate retention while controlling for performance during acquisition (Vnek, Kromer, Wiley, \& Rothblat, 1996), we calculated a savings score using the following formula: savings = (sessions to criterion acquisition - (sessions to criterion retention $) /$ sessions to criterion acquisition. A savings score of 0 indicates no savings; a score of 1 indicates perfect retention. The savings score for mice in Cohort 1 relearning Discrimination B after a retention interval of approximately 10 weeks was 0.73 ( \pm 0.03 ). After a retention interval of 1 week, the mean for mice in Cohort 2 relearning Discrimination C was $1.8( \pm 0.55)$ sessions, resulting in a savings score of $0.90( \pm 0.04)$. The mean number of correct responses on the first relearning session was 16.7 ( \pm 0.87 ), for an average accuracy score of $84 \%$.

\section{Discussion}

These results provide a clear demonstration that mice can successfully perform visual discriminations with computer-graphic stimuli. Moreover, it can be done with a behavioral protocol that is similar to that used for testing learning and memory in humans, monkeys, and rats. By using three different single-pair discriminations of varying difficulty, we found that all 8 mice studied were able to learn to discriminate such stimuli in this apparatus to a stringent criterion of $90 \%$ correct responses and that learning occurred with surprisingly little inter-animal variability. Mice can also be trained on more complex tasks, such as concurrentdiscrimination learning, that have been widely used as indicators of cognitive dysfunction in amnesic patients and animals with temporal lobe damage. In addition, after a retention interval was 
interposed between acquisition of the discrimination and a relearning test, mice showed evidence of substantial savings, even when the interval was filled with new discrimination training. Thus, it is possible to use discrete acquisition and retention phases in training subjects on lists of items (Vnek \& Rothblat, 1996), expanding the array of mnemonic variables that can be examined in transgenic and gene-knockout mouse models.

There are many advantages of testing mice using computerized methods. The automated touchscreen procedure not only reduces inadvertent experimenter bias but also greatly facilitates testing. Mice can complete a 20 -trial session within $15 \mathrm{~min}$, and animals can be run in separate test boxes simultaneously. The flexibility gained by using computer-controlled stimuli opens up the possibility of testing sensory capacity, attention, learning, and memory-both spatial and nonspatial-with the same apparatus. Thus, it is possible to manipulate a wide array of task demands for the purpose of obtaining comprehensive and reliable behavioral assays. Although such a method for mice would clearly be useful for drug and lesion studies, it would be particularly important for the behavioral characterization of transgenic and gene-knockout models. In many of these models, changes can occur not only in the brain but also in other parts of the nervous system, including, for example, the retina. For this reason, it is important to evaluate the extent to which putative cognitive deficits might be due to changes in basic sensory capabilities of the mouse. The use of computergraphic stimuli facilitates such assessment by allowing researchers to manipulate visual stimuli in a manner that could allow the systematic analysis of visual capacities, such as resolution acuity and contrast sensitivity.

Perhaps the most important benefit of using computer-graphic testing techniques for mice is that it allows for comparison of results with those of other species. Because visual discrimination tasks have been used for many years with monkeys and are now being used with rats, the development of discrimination procedures for mice provides the opportunity for more sophisticated comparative studies. Although further research is needed to characterize the similarities in cognitive processes and neural systems, it appears from initial findings that the performance of mice and rats when tested with the touch-screen apparatus is quite comparable (Bussey et al., 1994). It is interesting to note that Bussey et al. found that because of the innate impulsivity of the rat, good performance was obtained only when a shelf was positioned immediately below the stimuli, so that the rat could slow down, rear, place its paws on the shelf, and stretch across the shelf in order to make a nose poke toward the stimuli. In this study, we found that such additions were not required for mice. With the use of photocell touch-screen technology, the mice could easily respond to graphic stimuli presented on a VDU. When the stimuli were presented just above head height on a normal computer screen, in most cases, a mouse would approach the stimuli rapidly and then would slow down spontaneously before making a choice, often alternating glances between the two stimuli before nose-poking toward the chosen stimulus. Thus, it appears that the touchscreen testing method may be particularly well suited to the temperament of the mouse. The automated touchscreen procedure for the mouse may therefore provide a powerful new tool for exploring the neural mechanisms underlying cognition.

\section{References}

Barnes, C. (1979). Memory deficits associated with senescence: A neurophysiological and behavioral study in the rat. Journal of Comparative and Physiological Psychology, 93, 74-104.

Bussey, T. J., Muir, J. L., Everitt, B. J., \& Robbins, T. W. (1997). Triple dissociation of anterior cingulate, posterior cingulate, and medial frontal cortices on visual discrimination tasks using a touchscreen testing procedure for the rat. Behavioral Neuroscience, 111, 920-936.

Bussey, T. J., Muir, J. L., \& Robbins, T. W. (1994). A novel automated touchscreen procedure for assessing learning in the rat using computergraphic stimuli. Neuroscience Research Communications, 15, 103-110.

Bussey, T. J., Warburton, E. C., Aggleton, J. P., \& Muir, J. L. (1998). Fornix lesions can facilitate acquisition of the transverse patterning task: A challenge for "configural" theories of hippocampal function. Journal of Neuroscience, 18, 1622-1631.

Gaffan, E., \& Eacott, M. (1995). A computer-controlled maze environment for testing visual memory in the rat. Journal of Neuroscience Methods, 60, 23-37.

Hsiao, K., Chapman, P., Nilsen, S., Eckman, C., Harigaya, Y., Younkin, S., Yang, F., \& Cole, G. (1996, October 4). Correlative memory deficits. $\mathrm{A} \beta$ elevation, and amyloid plagues in transgenic mice. Science, 274, $99-102$.

Kim, J. J., \& Fanselow, M. S. (1992, May 1). Modality-specific retrograde amnesia of fear. Science, 256, 675-677.

LeDoux, J. E. (1992). Brain mechanisms of emotion and emotional learning. Current Opinions in Neurobiology, 2, 191-197.

Mayford, M., \& Kandel, E. R. (1999). Genetic approaches to memory. Trends in Genetics, 15, 463-470.

Morris, R. G. M. (1984). Development of a water maze procedure for studying spatial learning in the rat. Journal of Neuroscience Methods, $11,47-60$.

Rothblat, L. A., \& Hayes, L. L. (1987). Short-term object recognition memory in the rat: Nonmatching with trial-unique junk stimuli. Behavioral Neuroscience, 101, 587-590.

Rothblat, L. A., Schwartz, M. L., \& Kasdan, P. M. (1978). Monocular deprivation in the rat: Evidence for an age-related defect in visual behavior. Brain Research, 158, 456-460.

Rothblat, L. A., Vnek, N., Gleason, T. C., \& Kromer, L. F. (1993). Role of the parahippocampal region in spatial and non-spatial memory: Effects of parahippocampal lesions on rewarded alternation and concurrent object discrimination learning in the rat. Behavioural Brain Research, 55, 93-100.

Silva, A. J., Kogan, J. H., Frankland, P., \& Kida, S. (1998). CREB and memory. Annual Review of Neuroscience, 2l, 127-148.

Vnek, N., Kromer, L. F., Wiley, R. G., \& Rothblat, L. A. (1996). The basal forebrain cholinergic system and object memory in the rat. Brain Research, 710, 265-270.

Vnek, N., \& Rothblat, L. A. (1996). The hippocampus and long-term object memory in the rat. Journal of Neuroscience, 16, 2780-2787.

Received December 1, 2000 Revision received February 13, 2001 Accepted February 14, 2001 